\title{
Reinforced absorbent material: a cellulosic composite of TEMPO-oxidized MFC and CTMP fibres
}

\author{
Fredrik Wernersson Brodin • Kristoffer Lund • \\ Harald Brelid $\cdot$ Hans Theliander
}

Received: 9 January 2012/Accepted: 9 April 2012/Published online: 29 April 2012

(C) The Author(s) 2012. This article is published with open access at Springerlink.com

\begin{abstract}
This research aims to develop new materials based on renewable resources that can fulfill the functions necessary in the absorption core of a disposable diaper. Absorbent foam was recently produced from softwood kraft pulp by TEMPO oxidation, disintegration and freeze drying. In this study, the TEMPO-oxidized MFC was mixed with pulp fibres, thus forming a cellulosic composite, in an attempt to improve the mechanical stability of the freeze-dried absorbent material. The fibres were added in different amounts and the freeze-dried materials were evaluated for their absorption and retention properties. The results of this study suggest that the composite material has a better mechanical stability than the absorbent foam without fibres. It was shown that using spruce CTMP fibres in the composite resulted in better absorption and retention capacities than in a composite with softwood kraft pulp fibres. The higher stiffness of the CTMP fibres is a probable explanation for this difference. For the composite material with CTMP fibres, liquid porosimetry showed that pore size distribution was more or less retained when put under load. Furthermore, it was seen that the retention properties reached a maximum
\end{abstract}

F. W. Brodin · K. Lund · H. Brelid · H. Theliander $(\bowtie)$ Forest Products and Chemical Engineering, Department of Chemical and Biological Engineering, Chalmers University of Technology, SE-412 96 Gothenburg, Sweden

e-mail: hanst@chalmers.se around $85 \%$ CTMP fibres and $15 \%$ TEMPO-oxidized MFC. In the centrifuge retention test, the retention of the TEMPO-oxidized MFC in the composite material reached about the same capacity as conventional superabsorbent polymers.

Keywords Composite - Foam - TEMPO . CTMP fibres $\cdot$ MFC $\cdot$ Liquid retention

\section{Introduction}

Wood derived fibres are widely used in different products where the liquid absorption properties of fibre networks are utilized. These properties are at work in disposable diapers where so called fluff pulp fibres in combination with superabsorbent polymers (SAPs) form the absorbent core. In absorption articles, pulp fibres provide integrity to the absorption core. Furthermore, the fibres are important for the distribution of liquid and, to some extent, they also contribute to the absorption and retention of liquid. To supply these functions, a porous network with defined capillary structure is needed. This is accomplished by dry defibration of pulp and subsequent air-laying of the fibres in the absorption core. SAPs are used in absorbent articles because of their ability to absorb and retain large amounts of liquid (Nagorski 1994). The retention capacity of SAPs is dependent on, for example, degree of cross-linking and degree of protonization. Values for commercial SAPs in a 
centrifuge retention capacity test with $0.9 \% \mathrm{NaCl}$ solution are in the range of $28-37 \mathrm{~g} / \mathrm{g}$ (Nagorski 1994). During the air-laying of the absorbent core, SAP particles are mixed with fluff pulp. When this network of fibres with SAP particles is wetted, the SAPs attract water, and as a consequence, the SAP particles swell considerably. The SAP particle swelling drains the fibre network and immobilizes the liquid (Chatterjee 2002).

Since the introduction of SAPs in absorbent articles during the 1980s (Young and Barlow 2007), the composition of absorbent cores has changed. In a baby diaper, the amount of SAP was around $1 \mathrm{~g}$ in 1987, but increased to $13 \mathrm{~g}$ in 2005 (Edana 2007). At the same time, the total weight of the absorbent article has decreased by around $30 \%$. The main raw material in the production of SAP is currently based on petroleum, even though the first superabsorbents were based on renewable resources, such as cellulose and starch (Staples and Chatterjee 2002). The properties of modern SAPs that are, generally, based on crosslinked polyacrylates make them very efficient in applications such as diapers and feminine care products. Nevertheless, much research aimed at finding alternatives to conventional polyacrylate SAPs is ongoing. The motivation for this research is, to a large extent, related to decreasing dependence on petroleumbased products (Zohuriaan-Mehr and Kabiri 2008).

The present paper investigates the performance of microfibrillated cellulose (MFC) in absorbent applications. MFC, first developed by Herrick et al. (1983) and Turbak et al. (1983), is a material with interesting properties and it has been suggested for a wide range of usage areas (cf. e.g. Hubbe et al. 2008; Siró and Plackett 2010). One feature of MFC is that it forms gel-like structures when suspended in water. After freeze-drying the material obtained can be characterized as highly porous aerogels or foams (Pääkkö et al. 2008; Sehaqui et al. 2010; Saito et al. 2011; Sehaqui et al. 2011). MFC may for example be prepared after a TEMPO-oxidation pretreatment (Saito et al. 2006). In a recent study, a MFC-based absorbent material, in the form of a foam, was described (Theliander et al. 2011; Brodin and Theliander 2012). This foam was produced in a three-step process, including TEMPO-oxidation, disintegration and freeze drying. The retention properties of this material were about half of the capacity of conventional SAPs, but the rate of absorption was significantly faster. One reason for the lower retention capacity was the weak mechanical stability of the foam in the wet state.

In this study, an attempt has been made to produce a reinforced absorbent material with improved mechanical stability. By adding pulp fibres before the freeze drying step a cellulosic composite material was formed. Two different types of pulp fibres were evaluated; a softwood kraft pulp and a spruce chemithermomechanical pulp (CTMP). The fibres were added in different amounts and absorption and retention properties were studied.

\section{Experimental}

The composite material is based on two different cellulosic materials; a TEMPO-oxidized MFC and pulp fibres (either softwood kraft pulp or CTMP). The basic preparation steps are shown in Fig. 1.

\section{Materials}

The softwood chemical pulp was an industrial neverdried totally chlorine free bleached kraft pulp (peroxide-based bleach sequence) produced from Scandinavian softwood (a mixture of Picea abies and Pinus sylvestris). The brightness was $89 \%$ ISO. The CTMP was an industrial once-dried peroxide-bleached pulp produced from Norway spruce (Picea abies). The Canadian standard freeness (CSF) of the CTMP was $525 \mathrm{ml}$ and the brightness was $80 \%$ ISO.

Preparation of fibres

\section{Spruce CTMP}

The pulp was disintegrated in hot water at $3000 \mathrm{rpm}$ for 30000 revolutions (cf. SCAN-M 10:76), dewatered in a Büchner funnel, solvent-exchanged with acetone and air-dried.

\section{Softwood kraft pulp}

The softwood kraft pulp fibres were dried in the form of sheets (approx. $600 \mathrm{~g} / \mathrm{m}^{2}$ ) and dry defibrated in a hammer mill. A detailed procedure of the sheet forming has been described elsewhere (Lund et al. 2012). 
Fig. 1 Basic preparation steps for the composite material

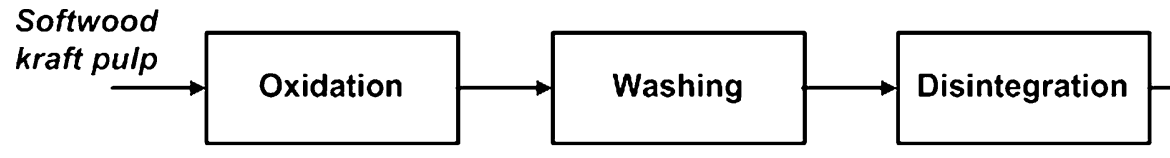

TEMPO-oxidized MFC

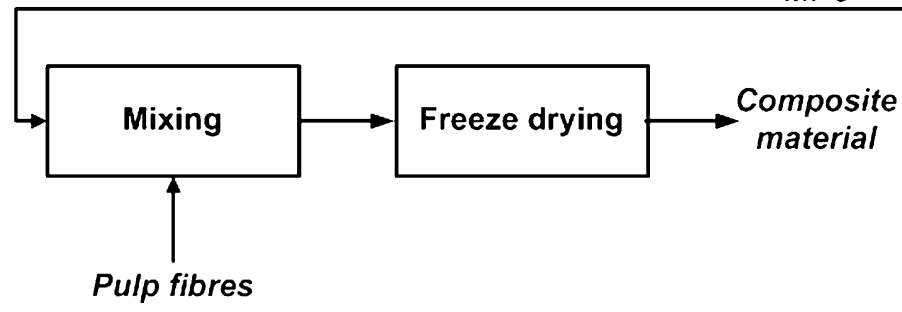

Preparation of TEMPO-oxidized MFC

The preparation of TEMPO-oxidized MFC has previously been described (Brodin and Theliander 2012). The two main steps in producing this material are oxidation and disintegration.

The softwood kraft pulp was oxidized in a reaction system containing $0.1 \mathrm{mM}$ TEMPO, $1 \mathrm{mM} \mathrm{NaBr}$ and $4.2 \mathrm{mmol} \mathrm{NaClO}$ per $\mathrm{g}$ of pulp. Four batches of pulp (12 $\mathrm{g}$ of pulp per batch, $1 \%$ pulp consistency) were oxidized at room temperature (approx. $21^{\circ} \mathrm{C}$ ) and washed. All four batches were mixed before disintegration. Batches of $3.0 \mathrm{~g}$ oxidized pulp were disintegrated for $9 \mathrm{~min}$ at $0.6 \%$ consistency using an Ultra-Turrax $\mathrm{T} 45 / \mathrm{N}$ (IKA). This material will be referred to as TEMPOoxidized MFC.

Preparation of the composites

\section{Mixing}

The fibres were mixed with the TEMPO-oxidized MFC in a glass beaker (inner diameter $=56 \mathrm{~mm}$ ) with an impeller (1450 rpm, impeller diameter $=50 \mathrm{~mm}$, four pitched blades). A fixed amount of TEMPO-oxidized MFC was used for each sample and the amount of fibres was varied. This gave materials with different dry content, and consequently, different test pad weights. Stirring was continued until a homogenous suspension was attained; which in this context means that no fibre bundles could be detected with ocular inspection.
Freeze drying

After the mixing step, the suspensions were freezedried (from dry contents of 0.6-5.0\% depending on fibre content). $20 \mathrm{~g}$ suspension was frozen in liquid nitrogen in a glass beaker (inner diameter $=47 \mathrm{~mm}$ ). During freezing, the glass beaker was surrounded by liquid nitrogen. The freeze drying was carried out using a Hetosicc CD 2.5 instrument operated at $0.3 \mathrm{mbar}$ for $48 \mathrm{~h}$. The dried test pads were stored in plastics bags at room conditions. The stored test pads had a dry content of around $95 \%$. The dried material with the addition of fibre will be referred to as composite material and the material without the addition of fibre will be referred to as foam material.

Analysis

\section{Fibre properties}

Fibre properties were analyzed with a Kajaani FS300. Duplicates were made for all samples. The pooled standard deviation was $0.10 \mathrm{~mm}$ for the fibre length, $1.6 \mu \mathrm{m}$ for the fibre width, $8 \mu \mathrm{g} / \mathrm{m}$ for the coarseness and $0.4 \%$ for the fines content. The fibre length, fibre width and fines content are reported as lengthweighted averages.

\section{Absorption properties}

The Free Swelling Capacity (FSC) and Centrifuge Retention Capacity (CRC) were measured according to Edana 440.1-99 and Edana 441.1-99. The FSC 
analysis involved soaking the samples in saline solution $(0.9 \% \mathrm{NaCl})$ at specified intervals and measuring their liquid uptake (by weight). CRC is the amount of liquid retained in a material after centrifugation. The methods deviated from the standard in two ways: the bags were allowed to drip off for 2 min before FSC weight measurements and the sample amount was $25 \%$ of a test pad $(0.03-0.25 \mathrm{~g})$. The results from these measurements are reported as mean values based on four samples. The relative standard errors were \pm $10 \%$ for FSC and $\pm 6 \%$ for CRC.

The wet bulk is an indirect measure of how much liquid can be retained in the material; a high wet bulk at a certain load indicates greater liquid retention. In the dry state, the bulk is the inverse of density. Wet bulk under load was measured in a compression tester. The equipment used for this analysis has been described previously (Lund et al. 2011). The freezedried samples were put in a compression tester and the height was measured at $0.6 \mathrm{kPa}$. The pressure was then released and an open liquid container was put under the sample. The sample absorbed liquid (water with 0.9 weight $\% \mathrm{NaCl}$ ) for $10 \mathrm{~min}$ followed by $2 \mathrm{~min}$ drainage. The pressure was then increased stepwise (as seen in Table 1) from 0.1 to $19.4 \mathrm{kPa}$ and after $2 \mathrm{~min}$ of equilibration at each pressure the height was measured. The wet bulk was calculated by dividing the sample volume by the dried weight of the test pad. The relative standard error of the wet bulk measurements was less than $\pm 5 \%$ at all pressures.

The CRC and wet bulk tests are two different ways to measure liquid retention, but the force acting on the

Table 1 The different pressures in the compression test

\begin{tabular}{lll}
\hline Time $(\mathrm{min})$ & Pressure $(\mathrm{kPa})$ & State \\
\hline 0 & 0.6 & Dry \\
2 & 0 & Wetting \\
12 & 0 & Drainage \\
14 & 0.1 & Wet \\
16 & 0.6 & Wet \\
18 & 1.3 & Wet \\
20 & 2.6 & Wet \\
22 & 5.2 & Wet \\
24 & 7.7 & Wet \\
26 & 12.9 & Wet \\
28 & 19.4 & Wet \\
\hline
\end{tabular}

The height was measured before each change in pressure samples are different. In the CRC test both the liquid and solid phases are affected by the forces during centrifugation, while in the wet bulk test the external load mainly affects the solid phase. Thus, the CRC value gives an indication of the amount of liquid stored in the capillary structure and the wet bulk is a test of the mechanical stability of the solid network.

\section{Scanning electron microscopy (SEM)}

Samples were prepared by cutting the dried material with a sharp scalpel. Prior to analysis, the samples were coated with a thin layer (approx. $10 \mathrm{~nm}$ ) of gold (JEOL JFC-1100E ion sputter). The microscope was a JEOL JSM-820 scanning microscope (voltage $20 \mathrm{kV}$ ).

\section{Liquid porosimetry}

The total cumulative volume (TCV) describes the amount of liquid that can be stored in a material in pores that are smaller than or equal to the pore radius of the measurement point, and can be used to measure pore volume distributions in fibrous networks. This is done by a step-wise removal of liquid from the sample pores. The equipment is described in e.g. Miller and Tyomkin (1994). In this investigation, the receding mode was used where the sample was pre-saturated prior to the measurements. The liquid used was water with the addition of a wetting agent (Triton X-100, $0.1 \%$ tenside in water solution). By using a wetting agent the material was considered to be totally wetted. The samples were tested with and without a load of $5.2 \mathrm{kPa}$.

\section{Results and discussion}

Properties of fibres and fibre test pads

Table 2 shows the individual fibre and fibre network properties of the softwood kraft pulp and the CTMP. CTMP is a high-yield pulp and most of the wood components remain in the fibre wall. This is reflected in the high coarseness of the pulp fibres. This is in contrast to the softwood kraft pulp where virtually all lignin and a significant part of the hemicelluloses are removed from the fibre walls during cooking and subsequent bleaching. One consequence of this is that CTMP fibres are generally stiffer than softwood kraft 
Table 2 Individual fibre and fibre network properties in CTMP and softwood kraft pulp (SKP)

\begin{tabular}{|c|c|c|c|c|c|c|c|}
\hline Pulp & Length (mm) & Width $(\mu \mathrm{m})$ & Coarseness $(\mu \mathrm{g} / \mathrm{m})$ & Fines $(\%)$ & FSC $(30 \mathrm{~min})(\mathrm{g} / \mathrm{g})$ & $\begin{array}{l}\text { Wet bulk } \\
(5.2 \mathrm{kPa})\left(\mathrm{cm}^{3} / \mathrm{g}\right)\end{array}$ & CRC $(\mathrm{g} / \mathrm{g})$ \\
\hline SKP & 2.69 & 28.3 & 216 & 0.8 & 20.9 & 6.7 & 1.5 \\
\hline CTMP & 2.13 & 38.7 & 348 & 3.1 & 26.4 & 8.6 & 2.2 \\
\hline
\end{tabular}

Length, width and fines are reported as length-weighted averages

pulp fibres. The effect of this can be seen in the FSC (Free Swelling Capacity) and in the wet bulk under load. Both of these properties are higher in the CTMP, which is likely because stiff fibres are more able to maintain a porous network. It can also be noted that the CRC (Centrifuge Retention Capacity) is higher in CTMP fibres. This may, at least partly, be explained by the higher content of fines in the material, which gives more available surfaces for liquid sorption.

\section{Properties of TEMPO-oxidized MFC}

and absorbent foam

The oxidation and disintegration treatment of the softwood kraft pulp fibres produced a TEMPOoxidized MFC that has a high content of functional groups. The TEMPO-oxidation treatment introduces both negatively charged carboxylate groups $\left(\mathrm{COO}^{-}\right)$ and reactive aldehyde groups $(\mathrm{CHO})$. The chemical and physical properties of the TEMPO-oxidized MFC and material properties of the foam after freeze drying have been presented in an earlier study (Brodin and Theliander 2012). In this study, the same raw material and the same method were used. Some of the relevant properties of the TEMPO-oxidized MFC and the foam are presented in Table 3.

Comparison between CTMP and softwood kraft pulp in the composite material

\section{SEM}

The foam material and two composite materials, with 85 weight- $\%$ fibres of either CTMP or softwood kraft pulp, were examined with SEM. In the three samples, the amount of TEMPO-oxidized MFC was equal. The low magnification images (Figs. 2a, 3a and 4a) show that the materials were homogenous and the fibres in the composite materials were evenly distributed. At higher magnification (Figs. $2 b, 3 b$ and $4 b$ ), the pores in the materials can be seen. In the foam material, the pores were divided by sheet-like layers connected to a fibrillar network. In the composite materials, the TEMPO-oxidized MFC was found between the fibres creating a narrow pore structure. Consequently, the relatively large capillaries between the fibres in a fibre network without TEMPO-oxidized MFC had been replaced by TEMPO-oxidized MFC with small capillaries. In Figs. 3b and 4b, TEMPO-oxidized MFC can also be seen connected to fibre surfaces and may possibly provide a more stable fibre network.

\section{Absorption properties}

The composite materials were tested for their absorption capacity (FSC). Futhermore, two different types of tests were used to investigate the liquid retention properties of the composite materials; CRC and wet bulk under load. It should also be noted that freeze drying of the materials resulted in a shrinkage of $10-15 \%$. Thus, the densities were between $7 \mathrm{mg} / \mathrm{cm}^{3}$ (at $0 \%$ fibre content) and $60 \mathrm{mg} / \mathrm{cm}^{3}$ (at $88 \%$ fibre content).

Table 4 shows a comparison between using CTMP and softwood kraft pulp in the composite material. The foam material, which does not include any fibres, and the two fibre types (CTMP and softwood kraft pulp)

Table 3 Functional groups and fibre length of the TEMPO-oxidized MFC and surface area and absorption properties of the absorbent foam (Brodin and Theliander 2012)

\begin{tabular}{lllllll}
\hline $\begin{array}{l}\mathrm{COO}^{-} \\
(\mathrm{mmol} / \mathrm{g})\end{array}$ & $\begin{array}{l}\mathrm{CHO} \\
(\mathrm{mmol} / \mathrm{g})\end{array}$ & $\begin{array}{l}\text { Fibre length } \\
(\mathrm{mm})\end{array}$ & $\begin{array}{l}\text { Surface area (BET) } \\
\left(\mathrm{m}^{2} / \mathrm{g}\right)\end{array}$ & $\begin{array}{l}\text { FSC (30 min) } \\
(\mathrm{g} / \mathrm{g})\end{array}$ & $\begin{array}{l}\text { Wet bulk (5.2 kPa) } \\
\left(\mathrm{cm}^{3} / \mathrm{g}\right)\end{array}$ \\
\hline 1.38 & 0.61 & 0.4 & 64.9 & 56.2 & 16.7 & 12.6 \\
\hline
\end{tabular}




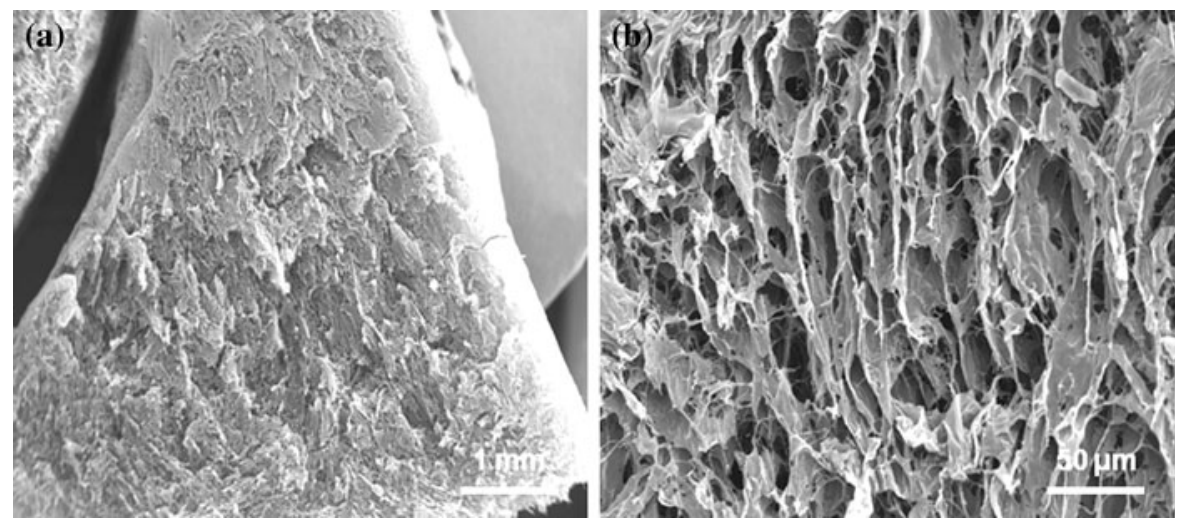

Fig. 2 SEM pictures of foam material at $\mathbf{a} \times 20$ and $\mathbf{b} \times 400$ magnification
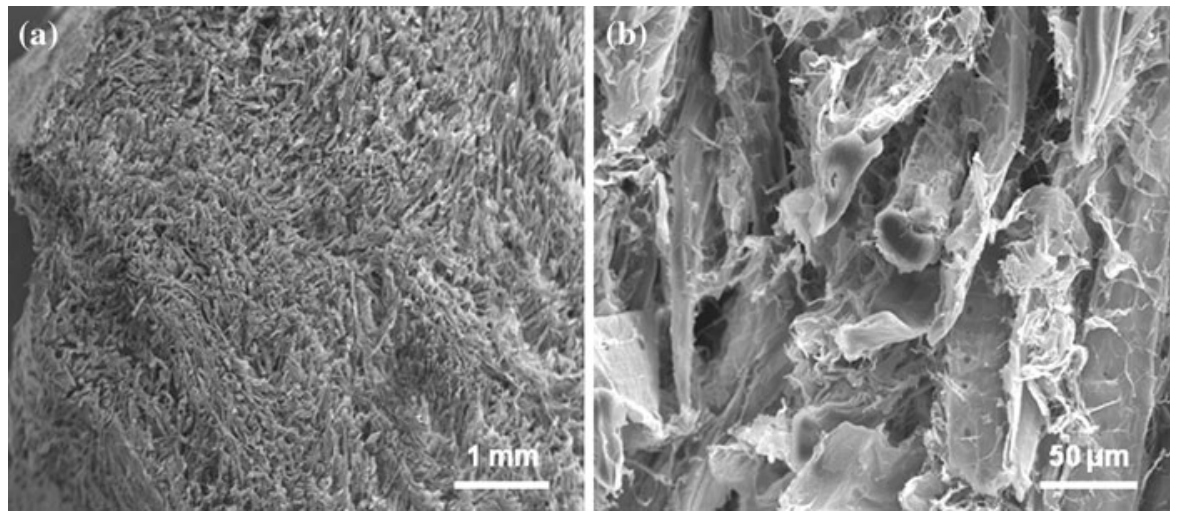

Fig. 3 SEM pictures of composite material with $85 \%$ softwood kraft pulp at $\mathbf{a} \times 20$ and $\mathbf{b} \times 400$ magnification

are also included in the table as references. It can be seen that when CTMP was used in the composite material, greater absorption and retention capacities were generally obtained than when softwood kraft pulp was used. The only exception is the CRC at fibre contents of 25 and $50 \%$ where a larger retention capacity was seen in the softwood kraft pulp. It is likely that the stiffer CTMP fibres preserve the porous structure of the network to a larger extent, especially under pressure.

The data in Table 4 shows that composite materials with CTMP fibres as reinforcing elements have better mechanical stability than the composite materials with softwood kraft pulp fibres. Therefore, a more comprehensive characterization of the composite materials with CTMP fibres was done. Additional fibre contents were evaluated to find the optimal mixture of TEMPOoxidized MFC and CTMP fibres. Comparisons were also made between foam, fibres and composite materials.
Evaluation of composite materials with CTMP fibres

\section{Liquid porosimetry}

The Total Cumulative Volume (TCV) and the Relative Total Cumulative Volume (RTCV) for the foam and the composite materials (with $85 \%$ CTMP fibres and $15 \%$ foam material) were analyzed with and without load $(5.2 \mathrm{kPa})$.

The initial TCV of the uncompressed foam material was $62.2 \mathrm{~cm}^{3} / \mathrm{g}$ (see Fig. 5a), which can be regarded as a measure of the maximum absorption capacity. The shape of the curve shows that the foam material has a relatively high content of large pores. This is in contrast with the uncompressed composite material, which mainly consists of smaller pores (small increase in TCV at large pore radii). The liquid removed from a sample at small pore radii was tightly bound in the material by strong capillary forces. 

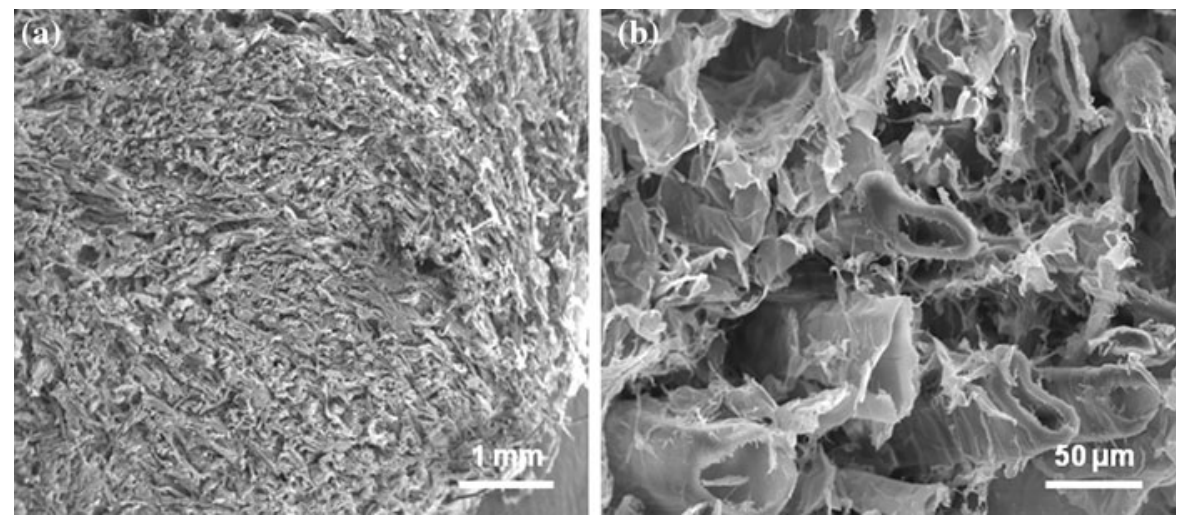

Fig. 4 SEM pictures of composite material with $85 \%$ CTMP at $\mathbf{a} \times 20$ and $\mathbf{b} \times 400$ magnification

Table 4 FSC, CRC and wet bulk under load for the composite material with CTMP and softwood kraft pulp (SKP) at different fibre contents

\begin{tabular}{lclrr}
\hline Pulp & $\begin{array}{l}\text { Fibre content } \\
(\%)\end{array}$ & $\begin{array}{l}\text { FSC }(30 \mathrm{~min}) \\
(\mathrm{g} / \mathrm{g})\end{array}$ & $\begin{array}{l}\text { CRC } \\
(\mathrm{g} / \mathrm{g})\end{array}$ & $\begin{array}{l}\text { Wet bulk (5.2 kPa) } \\
\left(\mathrm{cm}^{3} / \mathrm{g}\right)\end{array}$ \\
\hline Foam & 0 & 56.2 & 12.6 & 16.7 \\
CTMP & 100 & 26.4 & 2.2 & 8.6 \\
SKP & 100 & 20.9 & 1.5 & 6.7 \\
Composite (CTMP) & 25 & 57.4 & 10.9 & 17.0 \\
& 50 & 54.2 & 8.4 & 14.9 \\
& 75 & 50.4 & 7.0 & 15.0 \\
Composite (SKP) & 85 & 37.0 & 5.7 & 17.1 \\
& 25 & 55.5 & 12.9 & 16.5 \\
& 50 & 43.6 & 9.2 & 13.8 \\
\end{tabular}

The foam material and test pads of the two fibre types are included as references. Test liquid was $0.9 \% \mathrm{NaCl}$-solution

When these two materials were compressed under load, the initial TCV was less than in uncompressed materials. It was found that neither of the compressed materials contained a significant amount of large pores, and that mostly large pores in the foam material were compressed by the load (cf. Fig. 5a, b). In contrast to this, a rather similar RTCV was obtained for the composite material from both the compressed and uncompressed sample (cf. Fig. 5b), which means that the material had not collapsed according to pore size. This may be explained by effects of the fibre network in the composite material when it is compressed. Parts of the foam material attached to the fibres are compressed, while some parts are left intact (see Fig. 6). Consequently, the RTCV result suggests that the composite material has an improved mechanical stability in comparison with the foam material.

\section{Absorption capacity}

The FSC measurements showed absorption capacities after $30 \mathrm{~min}$ of $56 \mathrm{~g}$ liquid/g foam and $37 \mathrm{~g}$ liquid/g composite (15\% TEMPO-oxidized MFC/85\% CTMP fibres), cf. Fig. 7. The absorption capacity of the foam is on about the same level as conventional SAPs (cf. e.g. Mudiyanselage and Neckers 2007; Shimomura and Namba 1994). Given that the FSC after $1 \mathrm{~min}$ for the foam was already at $48.4 \mathrm{~g}$ liquid/g foam, the rate of absorption was rapid. This enables the material to quickly absorb liquid, which may be a 
desirable property in absorption applications. Similar trends were seen in the CTMP sample and the composite material. The absorption capacity results are consistent with the findings regarding pore volume distribution (cf. Fig. 5a). The foam material has a higher porosity and is thus able to absorb more liquid than the composite material in a free swelling test (FSC).
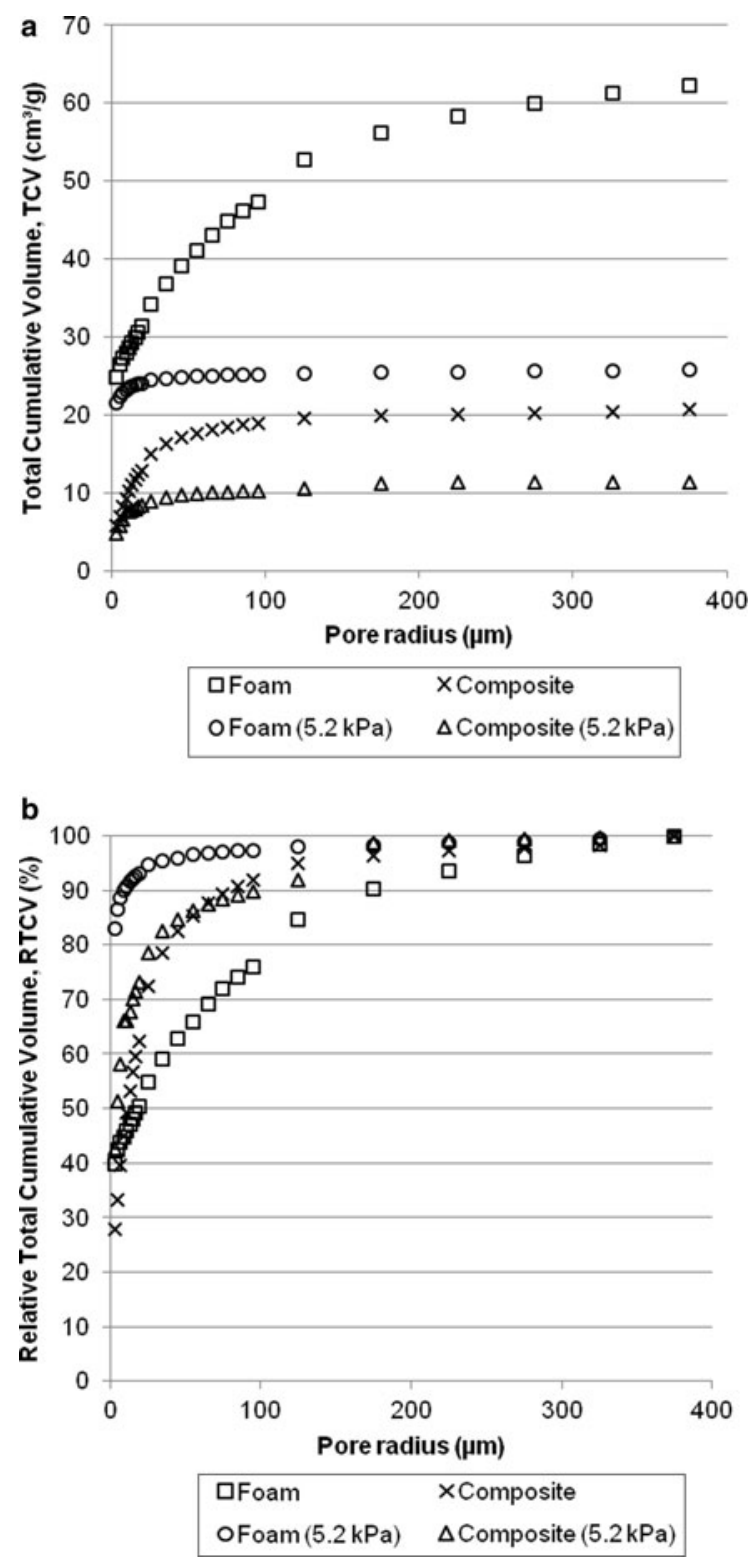

Fig. 5 a Total Cumulative Volume (TCV) and b Relative Total Cumulative Volume (RTCV) plotted against pore radius of the foam and the composite material (85\% CTMP fibres and $15 \%$ TEMPO-oxidized MFC)

\section{Liquid retention properties}

Results related to the liquid retention properties of the composite materials with different amounts of fibres can be seen in Fig. 8 (wet bulk under load) and Fig. 9 (CRC). Results obtained at three different pressures are shown for the wet bulk under load. The theoretical bulk is shown as a dotted line and represents the bulk obtained if the contributions from the foam and the CTMP fibres are considered separately. It is calculated by:

$$
\begin{aligned}
\text { Theoretical bulk }= & \mathrm{x}_{\text {Foam }} \times \text { Wet bulk } \\
& \times \text { Weam bulk } \mathrm{K}_{\mathrm{CTMP}}+\mathrm{x}_{\mathrm{CTMP}} \\
& \times \text { We }
\end{aligned}
$$

where $\mathrm{x}$ is the mass fraction of each material. It can be seen that the composite material performs better than the theoretical bulk at low pressure $(0.5 \mathrm{kPa})$, especially at fibre contents around $50 \%$. This effect can also be seen at higher pressures. Nevertheless, at the higher pressures the results indicate a maximum gain in wet bulk (in comparison to the theoretical wet bulk) at approx. $85 \%$ fibre content. Thus, at low loads only a small number of fibres are required to stabilize the network in the composite material, while at higher pressures more fibres are needed to achieve the same result. It is likely that this is an effect of fibres reducing the load on the foam material. This effect thereby enables the TEMPO-oxidized MFC to retain more of its liquid retention properties when subjected to a load. An enhanced wet bulk was also obtained when softwood kraft pulp fibres were used, but as seen in Table 2, not to the same extent.

The results of the CRC measurements showed results similar to the ones for the wet bulk (cf. Figs. 8 and 9. The theoretical CRC was calculated in the same way as the theoretical bulk (cf. Eq. 1). CTMP fibres have a poor ability to retain liquid with a CRC of $2.2 \mathrm{~g}$ saline solution/g dried material, and consequently there is a lower CRC in the composite materials than in the foam (see Fig. 9). However, the values are consistently greater than the theoretical CRC and the effect is, similar to the wet bulk at higher pressures, more pronounced when fibre contents exceed $50 \%$.

The additional liquid (the difference between CRC and the theoretical CRC) that is retained in the composite material can be considered to be stored in the TEMPO-oxidized MFC, and in between the 
Fig. 6 Schematic illustration comparing an uncompressed and a compressed fibre network and how this affects the TEMPO-oxidized MFC depending on the location in the fibre network

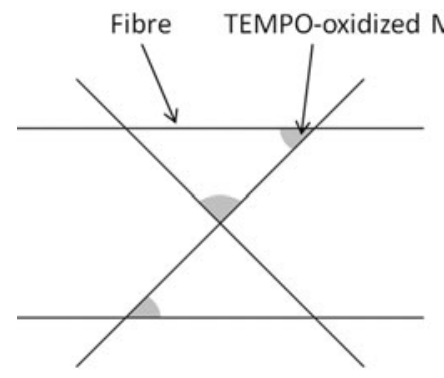

Composite material is compressed

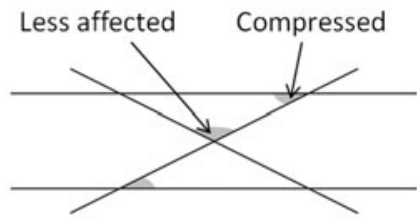

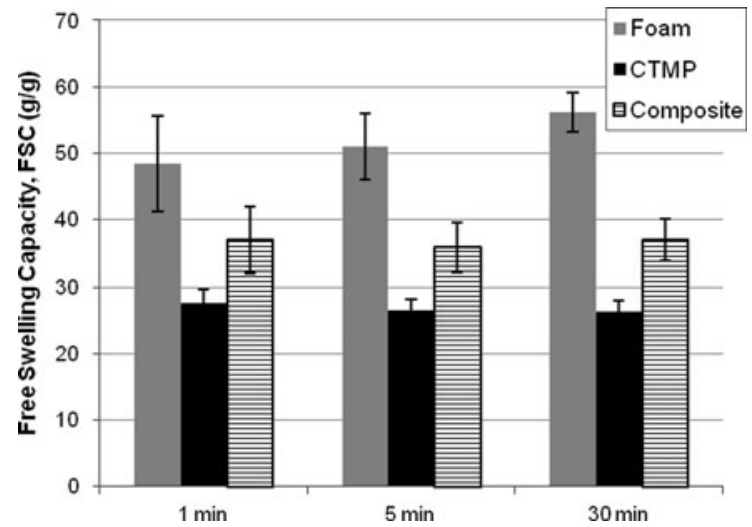

Fig. 7 FSC after 1, 5 and 30 min for the foam, the CTMP fibres and the composite material (85\% CTMP fibres and $15 \%$ TEMPO-oxidized MFC). Test liquid was $0.9 \% \mathrm{NaCl}$-solution. Error bars show the standard deviation of the measurements

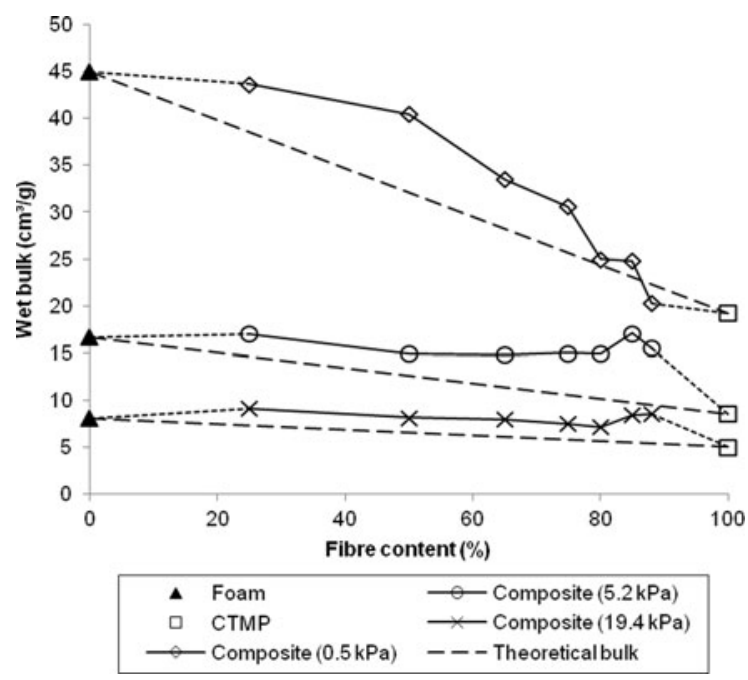

Fig. 8 The wet bulk under load for different fibre contents in the composite at three different pressures. The theoretical bulk was obtained by considering the contributions from the foam and the CTMP fibres separately (cf. Eq. 1). Test liquid was $0.9 \% \mathrm{NaCl}$-solution

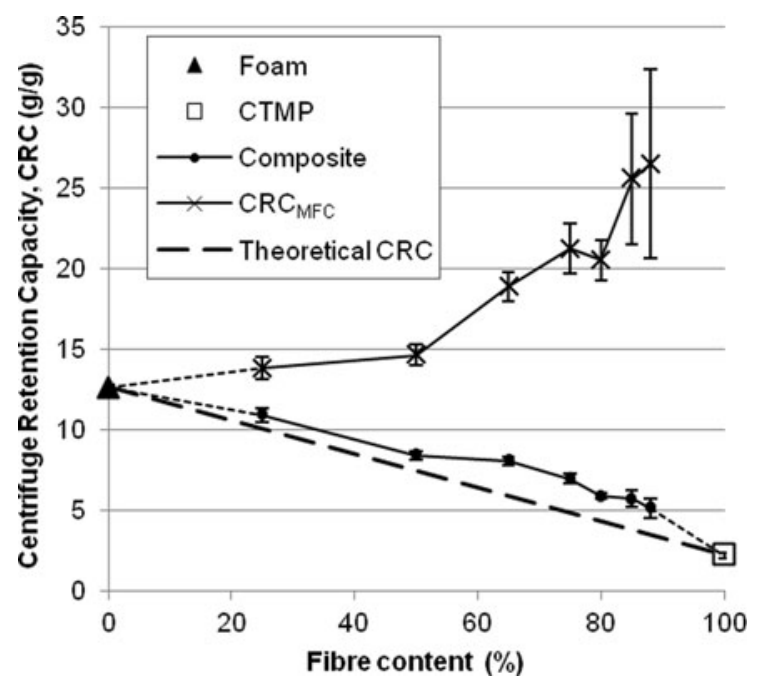

Fig. $9 \mathrm{CRC}$ and $\mathrm{CRC}_{\mathrm{MFC}}$ values for the composite material at different fibre contents as well as CRC for the foam and fibre references. $\mathrm{CRC}_{\mathrm{MFC}}$ was calculated according to Eq. 2. The theoretical CRC was obtained by considering the contributions from the foam and the CTMP fibres separately. Error bars represent the standard deviation of the measurements. Test liquid was $0.9 \% \mathrm{NaCl}$-solution

TEMPO-oxidized MFC and the fibre surfaces. If the additional liquid is entirely contributed to the TEMPO-oxidized MFC, a new CRC value can be obtained with a mass balance equation according to:

$$
\begin{aligned}
\mathrm{CRC}_{\mathrm{MFC}}= & \left(\mathrm{CRC}_{\text {Composite }} \times \mathrm{m}_{\text {Composite }}-\mathrm{CRC}_{\mathrm{CTMP}}\right. \\
& \left.\times \mathrm{m}_{\mathrm{CTMP}}\right) / \mathrm{m}_{\mathrm{MFC}}
\end{aligned}
$$

Fibre addition shows a positive effect on $\mathrm{CRC}_{\mathrm{MFC}}$ and the trend is that increasing the fibre content results in greater retention capacities of the TEMPO-oxidized MFC. Consequently, these findings indicate that more liquid was retained in the TEMPO-oxidized MFC when it was combined with fibres. However, it should be noted that the standard deviations are greater for the 
$\mathrm{CRC}_{\mathrm{MFC}}$ than for the $\mathrm{CRC}$ of the composite material. The $\mathrm{CRC}_{\mathrm{MFC}}$ is on about the same level as the SAPs, which have a CRC of about $30 \mathrm{~g} / \mathrm{g}$ (cf. e.g. Nagorski 1994).

\section{Application perspective}

The absorption core of disposable diapers contains a mixture of fluff pulp fibres and SAPs. The mixture is different depending on product requirements. Fluff pulp fibres give integrity to the network and distribute liquid throughout the product, while the main function of SAPs is to retain liquid. The composite material absorbs liquid rapidly and it has an enhanced ability to retain liquid in comparison to a network containing only fluff pulp fibres. Thus, it may be possible to, at least partly, replace the oil-based materials in the absorbent core of a disposable diaper with cellulosic material and maintain product performance.

\section{Conclusions}

It has been shown that addition of fibres has a positive effect on the retention properties of the TEMPOoxidized MFC produced from softwood kraft pulp. This effect was more pronounced if CTMP fibres were used in the composite material, instead of softwood kraft pulp. It is likely that this is due to the higher fibre stiffness of CTMP fibres. In a more in-depth study of the composite with CTMP fibres it was found that the RTCV was similar for the compressed material and the uncompressed material, while the foam material had a large decrease in large pores. This means that pore size distribution is relatively unaffected in the composite material, even though the TCV has decreased. This suggests that the composite material had improved mechanical stability. Furthermore, the retention properties of the composite material were better than the theoretical retention properties. A maximum in retention properties was also seen at fibre contents of around $85 \%$. At these fibre contents, the centrifuge retention capacity of the TEMPO-oxidized MFC was comparable with the capacity of commercial SAPs (26.5 g/g for the TEMPO-oxidized MFC compared to around $30 \mathrm{~g} / \mathrm{g}$ for commercial SAPs). However, due to the low retention capacity of the CTMP fibres, the centrifuge retention capacity of the composite material was lower than that of the foam material.
Acknowledgments The authors would like to express gratitude to "Södra Skogsägarnas stiftelse för forskning, utveckling och utbildning", SCA Hygiene Products AB and VINNOVA for financial support in the project. Maria Fernkvist at SCA Hygiene Products AB is gratefully acknowledged for help with practical work and rewarding discussions.

Open Access This article is distributed under the terms of the Creative Commons Attribution License which permits any use, distribution, and reproduction in any medium, provided the original author(s) and the source are credited.

\section{References}

Brodin F, Theliander H (2012) Absorbent materials based on kraft pulp: preparation and material characterization. Bioresources 7(2):1666-1683

Chatterjee P (2002) Products and technology perspective. In: Chatterjee P, Gupta B (eds) Absorbent technology. Elsevier Science B.V, Amsterdam, p 460

Edana (2007) Sustainability report 2007-2008: absorbent hygiene products. URL: http://www.edana.org

Herrick F, Casebier R, Hamilton J, Sandberg K (1983) Microfibrillated cellulose: morphology and accessibility. J Appl Polym Sci: Appl Polym Symp 37:797-813

Hubbe M, Rojas O, Lucia L, Sain M (2008) Cellulose nanocomposites: a review. Bioresources 3(3):929-980

Lund K, de la Motte H, Brelid H, Westman G, Hanson C (2011) Diepoxide treatment of softwood kraft pulp: influence on absorption properties of fibre networks. Cellulose 18(5): $1365-1375$

Lund K, Sjöström, K, Brelid H (2012) Alkali extraction of kraft pulp fibres: Influence on fibre and fluff pulp properties. J Eng Fiber Fabr 7(3)

Miller B, Tyomkin I (1994) Liquid porosimetry: new methodology and applications. J Colloid Interface Sci 162: 163-170

Mudiyanselage T, Neckers D (2007) Highly absorbing superabsorbent polymer. J Polym Sci, Part A: Polym Chem 46(7):1357-1364

Nagorski H (1994) Characterization of a new superabsorbent polymer generation. In: Buchholz F, Peppas N (eds) Superabsorbent technology: Science and technology. American chemical society, Washington, pp 101-103

Pääkkö M, Vapaavuori J, Silvennoinen R, Kosonen H, Ankerfors M, Lindström T, Berglund LA, Ikkala O (2008) Long and entangled native cellulose I nanofibers allow flexible aerogels and hierarchically porous templates for functionalities. Soft Matter 4:2492-2499

Saito T, Nishiyama Y, Putaux J-L, Vignon M, Isogai A (2006) Homogenous suspensions of individualized microfibrils from TEMPO-catalyzed oxidation of native cellulose. Biomacromolecules 7(6):1687-1691

Saito T, Uematsu T, Kimura S, Enomae T, Isogai A (2011) Selfaligned integration of native cellulose nanofibrils towards producing diverse bulk materials. Soft Matter 7:8804-8809

Sehaqui H, Salajková M, Zhou Q, Berglund L (2010) Mechanical performance tailoring of tough ultra-high 
porosity foams prepared from cellulose I nanofiber suspensions. Soft Matter 6:1824-1832

Sehaqui H, Zhou Q, Berglund L (2011) High-porosity aerogels of high specific surface area prepared from nanofibrillated cellulose (NFC). Compos Sci Technol 71:1593-1599

Shimomura T, Namba T (1994) Preparation and application of high-performance superabsorbent polymers. In: Buchholz F, Peppas N (eds) Superabsorbent technology: Science and technology. American chemical society, Washington, pp 113-114

Siró I, Plackett D (2010) Microfibrillated cellulose and new nanocomposite materials: a review. Cellulose 17(3):459494

Staples T, Chatterjee P (2002) Synthetic superabsorbents. In: Chatterjee P, Gupta B (eds) Absorbent technology. Elsevier Science B.V, Amsterdam, p 284
Theliander H, Wernersson F, Hansson C, Gustafson I, Falk T (2011) Absorbent article comprising absorbent porous foam of freeze-dried microfibrillated cellulose. WO Patent 2011090410, 28 July 2011

Turbak A, Snyder F, Sandberg K (1983) Microfibrillated cellulose, a new cellulose product: properties, uses, and commercial potential. J Appl Polym Sci: Appl Polym Symp 37:815-827

Young D, Barlow F (2007) Past, present and future of the global fluff pulp market. Nonwovens world 16(1):51-56

Zohuriaan-Mehr M, Kabiri K (2008) Superabsorbent polymer materials: a review. Iran Polym J 17(6):451-477 